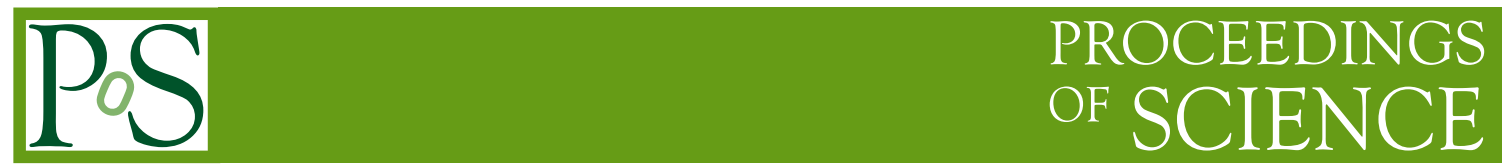

\title{
Heavy quarks and quarkonia production in high-energy experiments
}

\section{Sandro Palestini}

CERN, CH 1211 Geneva 23, Switzerland

E-mail: sandro.palestini@cern.ch

Proton-proton collisions at the LHC provide the ground for tests of the strong interactions through the study of the production mechanisms for quarkonia and hadrons containing charm or beauty flavours. This review addresses recent results and includes studies of kinematical correlations, polarisation and associated production. QCD-based models are briefly discussed and compared to measurements.

XVII International Conference on Hadron Spectroscopy and Structure - Hadron 2017 25-29 September, 2017

University of Salamanca, Salamanca, Spain 


\section{Introduction}

The remarkable magnitudes of collision energy and integrated luminosity achieved in protonproton collisions at the LHC have opened the possibility of detailed tests of QCD through studies of the production of hadron containing heavy quarks and of quarkonia. After brief introduction to the theory framework, the following sections discuss results on the production of $c$ and $b$ hadrons, on $b-\bar{b}$ kinematical correlations, on quarkonia $(c \bar{c}$ and $b \bar{b})$ production, on quarkonia polarisation, and on the associated production of $J / \psi$ pairs.

\section{Production of hadrons containing heavy quarks in hadron collisions}

The formal description of the production cross-section of a hadron $H_{Q}(Q=c, b)$ in highenergy hadron collisions is based on the factorisation formula

$$
\frac{d \sigma^{Q}}{d X}=\sum_{i, j} \int d \hat{X} \int d x_{1} \int d x_{2} f_{i}\left(x_{1}, q\right) f_{j}^{\prime}\left(x_{2}, q\right) \frac{d \hat{\sigma}_{i, j}^{Q}\left(x_{1}, x_{2}, s, q\right)}{d \hat{X}} F(\hat{X}, X, q),
$$

where the final states is specified by the set of variables $X$, and the process is described as the stronginteraction scattering of partons $i, j$, with Feynman variables $x_{1}, x_{2}$, into the state $\hat{X}$, integrated over the parton distributions (PDFs) $f_{i}, f_{j}{ }^{\prime}$ of the colliding hadrons, with the fragmentation function $F$ describing the transition to the final state, particle-level variables. The PDF's are universal and are extracted from fits to processes where the parton scattering is known with high accuracy. Similarly, the function $F$ includes long-distance effects, and can be extracted from independent observations (e.g. $b$-hadron fragmentation in $e^{+} e^{-} \rightarrow Z \rightarrow b \bar{b}$ ).

All terms in the factorisation equation depend logarithmically on energy scales, represented by $q$ in equation 2.1. Besides the factorisation scale, which affects the PDF's, and the renormalisation scale of the strong coupling $\alpha_{s}$, which enters the parton cross-section, the production of heavy quarks depends also on the scales set by the heavy quark mass $\left(m_{Q}\right)$ and by the transverse momentum $\left(p_{\mathrm{T}}\right)$ of the hadron $H_{Q}$.

Different ranges of $p_{\mathrm{T}}$ imply different computational methods. For values $p_{\mathrm{T}} \lesssim 5 \times m_{Q}$, only light flavours are included as active constituents $(u, d, s$ and also $c$ for beauty production, together with the gluon $g$ ) in the Fixed-Flavour-Number-Scheme (FFNS). For larger values of $p_{\mathrm{T}}$, the logarithm $\ln \left(p_{\mathrm{T}} / m_{Q}\right)$ is large and requires resummation, the heavy quarks are treated as active partons (Variable-Flavour-Number-Scheme VFNS), and all quarks may be treated as massless (Zero-Mass VFNS). In the fragmentation function $F$, a scale-dependent pertubative term may be combined with the scale independent term determined from fits to data.

Unified frameworks merging the low $p_{\mathrm{T}}$, massive computation and the high $p_{\mathrm{T}}$, massless computation have been developed, in particular the General-Mass VFNS (GM-VFNS) [1] and the Fixed-Order plus Next-to-Leading-Logarithms (FONLL) [2] schemes. Both methods rely on perturbative computations performed at Next-to-Leading-Order (NLO) and Next-to-LeadingLogarithms (NLL). Besides uncertainties related to the limited perturbative sums, the validities of these computation is related to assumptions in the transitions between different regimes, and in the use of non-perturbative fragmentation functions. Recent experimental results, discussed below, 


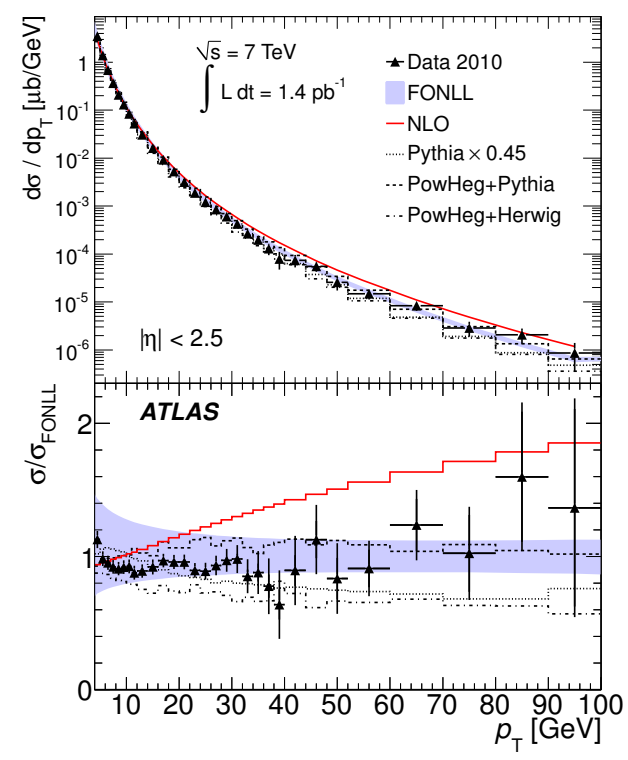

Figure 1: Muon differential cross-section for charm and beauty semileptonic decays, vs. muon $p_{\mathrm{T}}$. From reference [8].

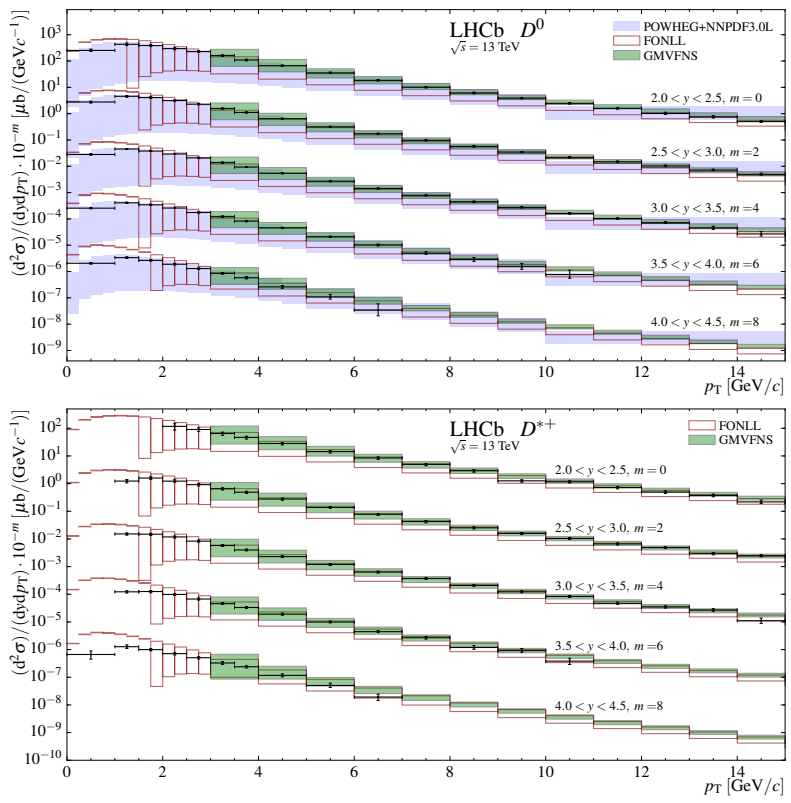

Figure 2: $D^{o}, D^{*+}$ production in the forward region. From reference [9].

have extended the test of the prediction to larger ranges in $p_{\mathrm{T}}$ and rapidity $y$, and to higher collision energies $\sqrt{s}$.

The experimental observables are frequently compared to Monte Carlo generators designed to model the production mechanism and the hadronisation process. PYTHIA [3] and HERWIG [4] describe the parton scattering at leading order (LO) and leading logarithm (LL) approximation, but NLO modelling has become available with the development of MC@NLO [5], POWHEG [6] and MADGRAPH [7].

\subsection{Measurements of charm and beauty production}

The production of hadrons containing heavy quarks may be studied using fully reconstructed final states, or with partially reconstructed final states. An example of the latter approach is shown in figure 1 , where the $p_{\mathrm{T}}$ spectrum of muons for pseudo-rapidity $|\eta|<2.5$ is provided by ATLAS [8] after subtracting the contribution from Drell-Yan continuum and $W, Z$ decays. Semileptonic decays of charm and beauty hadrons contribute to the spectrum. The data are compared to predictions and found in reasonable agreement, in particular with FONLL. The spectrum indicated with NLO shows the effect of removing the NLL component in FONLL.

LHCb has studied beauty and charm production in the forward, low $p_{\mathrm{T}}$ region. Figure 2 shows the measurement of $D^{o}$ and $D^{*+}$ mesons, with $D^{+}$and $D_{s}^{+}$and also presented in reference [9]. While usually the measurement of heavy-quark production cross-section is used to test the shortdistance parton scattering and not the parton PDFs, the ratio between the measurements at 13 and $7 \mathrm{TeV}$ in the forward region reaches the sensitivity for a test of the gluon distribution function [10].

The production cross-section of $B^{+}$has been measured in the central rapidity region $(|y| \lesssim 2.2)$ by CMS [11] and ATLAS [12] in the decay to $J / \psi K^{+} \rightarrow \mu^{+} \mu^{-} K^{+}$, with the muon pair used for 

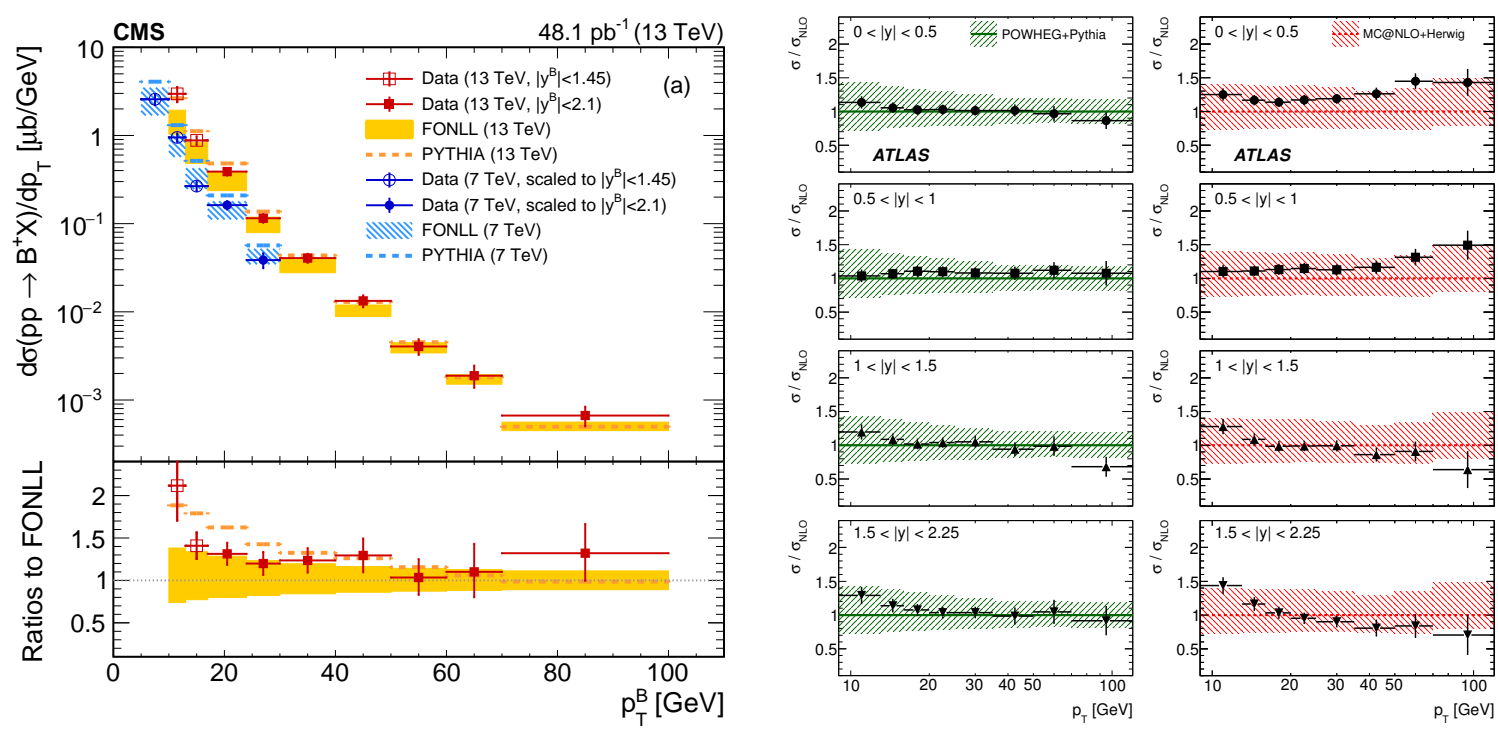

Figure 3: $B^{+}$production cross-section as a function of $p_{\mathrm{T}}$ in the central $y$ region, at 7 and $13 \mathrm{TeV}(l e f t)$, and for different $y$ intervals at $7 \mathrm{TeV}(r i g h t)$. From references [11] and [12].

on-line selection. Figure 3 shows good agreement with FONLL and POWHEG for $p_{\mathrm{T}}>15 \mathrm{GeV}$. Figure 4 shows the measurement of LHCb in the forward region [13], where the dependence on the rapidity is only in fair agreement with FONLL, which instead describes nicely the increase in production rate between 7 and $13 \mathrm{TeV}$. Production cross-sections have been measured also for $\Lambda_{b}^{o}[14,15], B^{o}$ [15], and for the ratio of production cross-section times branching-fraction to final state between $B_{c}^{+} \rightarrow J / \psi \pi^{+}$and $B^{+} \rightarrow J / \psi K^{+}[16,17]$.

\subsection{Correlations in $b-\bar{b}$ production}

The kinematical correlations between $b$ - and $\bar{b}$-hadrons have recently attracted interest. At the LHC energies, the $t$ channel $g g \rightarrow b \bar{b}$ process dominates the LO contribution, while at NLO the gluon splitting $g \rightarrow b \bar{b}$ is relevant at high $p_{\mathrm{T}}$ and for small opening angle between the two hadrons.
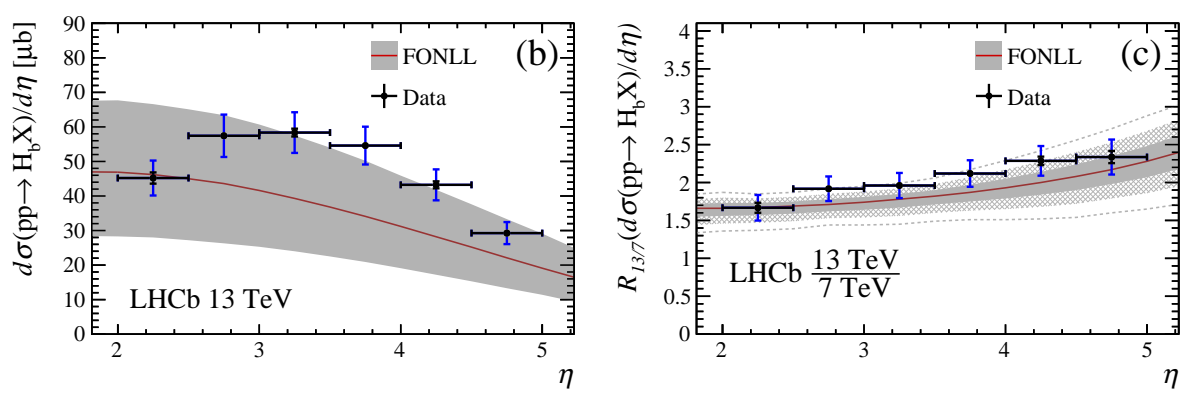

Figure 4: Rapidity dependence of the $b$-hadron production cross-section and of the ratio of the cross-section at 13 and $7 \mathrm{TeV}$, in the forward region. From reference [13]. 

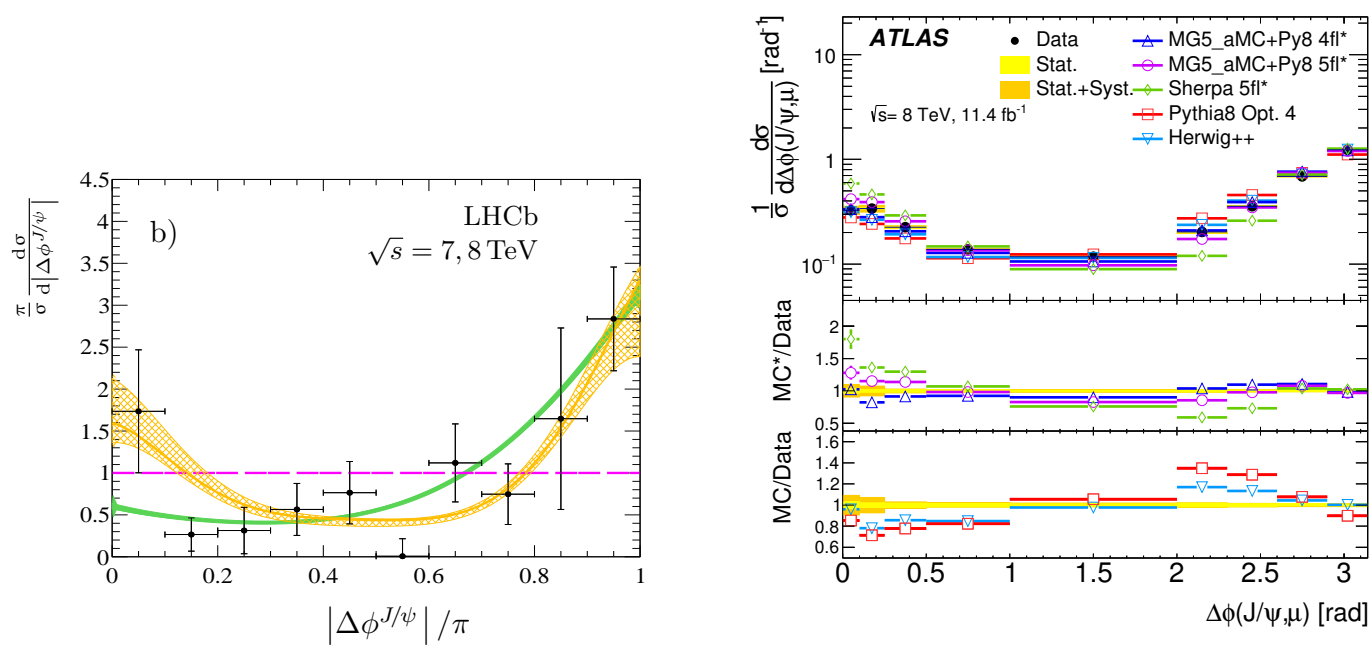

Figure 5: Left: azimuthal angular separation between $J / \psi$ 's from $b, \bar{b}$ in the forward region, with $p_{\mathrm{T}}{ }^{J / \psi}>$ $7 \mathrm{GeV}$. The yellow (green) band shows the POWHEG (PYTHIA) prediction. From reference [18]. Right: azimuthal angles separation between $J / \psi\left(\mu^{+} \mu^{-}\right)$and $\mu^{ \pm}$from $b, \bar{b}$ in the central region, with $p_{\mathrm{T}}{ }^{\mu}>6 \mathrm{GeV}$, compared to predictions. From reference [19].

Figure 5-left shows a recent result from LHCb [18], based on pairs of non-prompt $J / \psi$ 's, with the data favouring the NLO enhancement at small difference in azimuthal angle. The expected enhancement is more evident in the data collected by ATLAS [19], looking at non-prompt $J / \psi$ and $\mu^{ \pm}$, in a region of higher $p_{\mathrm{T}}$, as shown in figure 5-right. NLO computations, in particular MADGRAPH5_aMC+PYTHIA/4-flavours, provide a rather accurate description of the data.

\section{Quarkonia production}

The prompt production of hidden heavy flavour, i.e. of quarkonia $c \bar{c}, b \bar{b}$ bound states, differs from the production of open heavy flavour, since long-distance, non-perturbative effects enter the process more directly than the quasi-universal fragmentation fraction $F$ of equation 2.1.

The Colour Evaporation Model (CEM) [20, 21] relates the production cross-section of quarkonia to the production of open heavy flavour, in the limit of zero relative momentum and with the invariant mass of the $Q \bar{Q}$ pair below the threshold for hadron pair production $\left(2 \times m_{Q}<m_{Q \bar{Q}}<\right.$ $\left.2 \times m\left(H_{Q}\right)\right)$. Figure 6-left shows fair agreement between CEM and observed differential crosssection for $J / \psi[22]$.

The Colour Singlet Model (CSM) [23] assumes that a pertubative computation should be made including in the parton scattering only diagrams where the $Q \bar{Q}$ pair is produced in a colour-singlet configuration. The model has predictive powers since the only required external input is the amplitude of the wave function of the $Q \bar{Q}$ system at zero separation $\left(|\Psi(r=0)|^{2}\right)$, which can be extracted from decay widths. NLO and partial next-to-next leading order (NNLO*) computation [24] have been found to modify significantly the predicted cross-section, as shown in Figure 6-left.

The Non-Relativistic QCD (NRQCD) [25] approach describes quarkonia production in terms of an effective theory, where long-distance effects are treated with non-perturbative matrix elements 

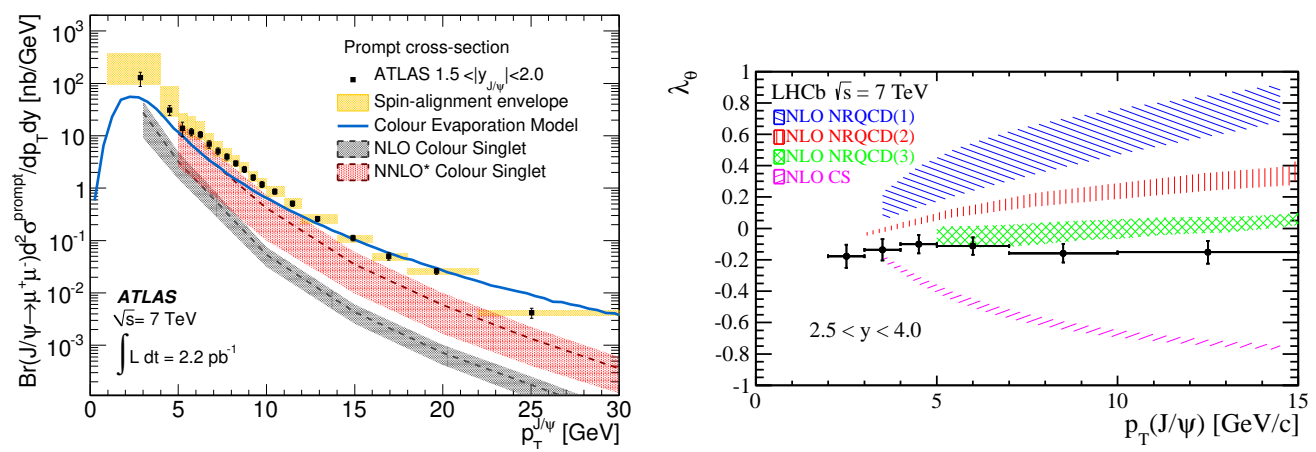

Figure 6: Transverse momentum dependence of $J / \psi$ production cross-section (left) and spin-alignment parameter $\lambda_{\theta}(r i g h t)$, compared to models. From references [22] and [39].

(LDME). In this scheme $Q \bar{Q}$ pairs produced in a coloured state (colour-octet) are included in the computation, assuming a transition into colourless physical states through the emission of soft gluons.

\subsection{Measurements of quarkonia production}

Measurements of quarkonia production have been performed at the LHC for all available collision energies. Detailed studies have been performed recently on vector states $(J / \psi, \psi(2 \mathrm{~S}), \Upsilon(\mathrm{nS})$, $\mathrm{n}=1,2,3)$ decaying to muon pairs [26, 27, 28, 29]. Measurements of $\psi(2 \mathrm{~S}) \rightarrow J / \psi \pi^{+} \pi^{-}$[30], $\chi_{c} \rightarrow J / \psi \gamma[31], \chi_{b} \rightarrow \Upsilon \gamma[32,33]$ and $\eta_{c}(1 S) \rightarrow p \bar{p}$ [34] are also available. For charmonium, the distinction between prompt and non-prompt production from $b$-hadron decays is obtained from a fit to the distance between the $p p$ interaction vertex and the muon-pair secondary vertex, which reflects the relatively long $b$-hadron lifetime and allows for an accurate separation. This technique, which is also used for the selection of $b$ - and $c$-hadrons from background events, is illustrated in figure 7. Conversely, feed-down from higher mass quarkonia state (e.g. $\chi_{c(b)}$ to $J / \psi(\Upsilon)$ transitions) is usually not subtracted from the experimental data, but can be evaluated from cross-section measurements and known branching fractions.
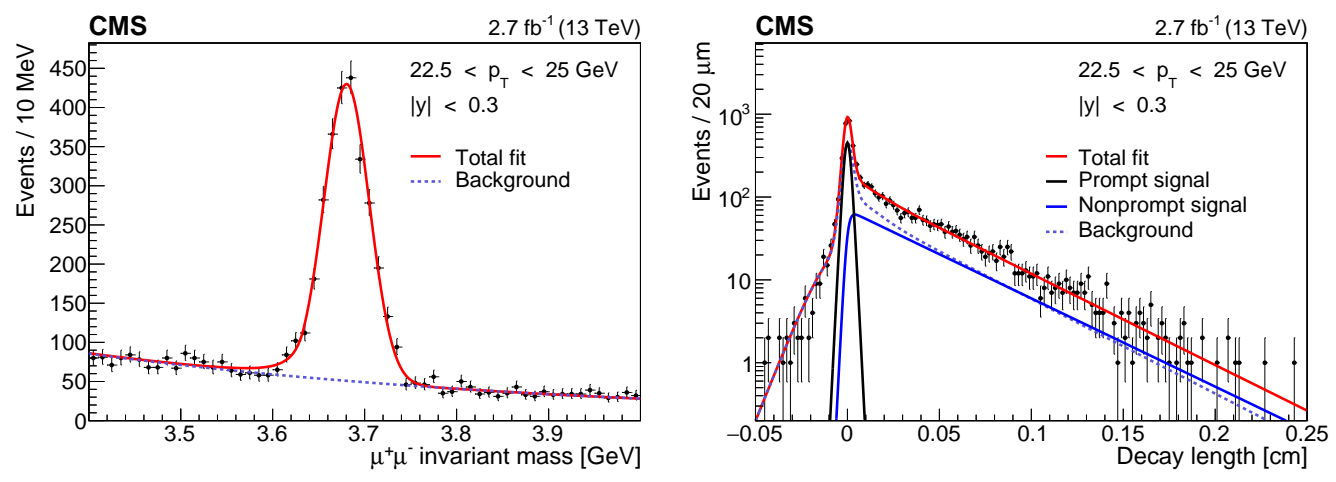

Figure 7: Simultaneous fit to invariant mass and vertex separation for $\psi(2 S) \rightarrow \mu^{+} \mu^{-}$. From reference [29]. 

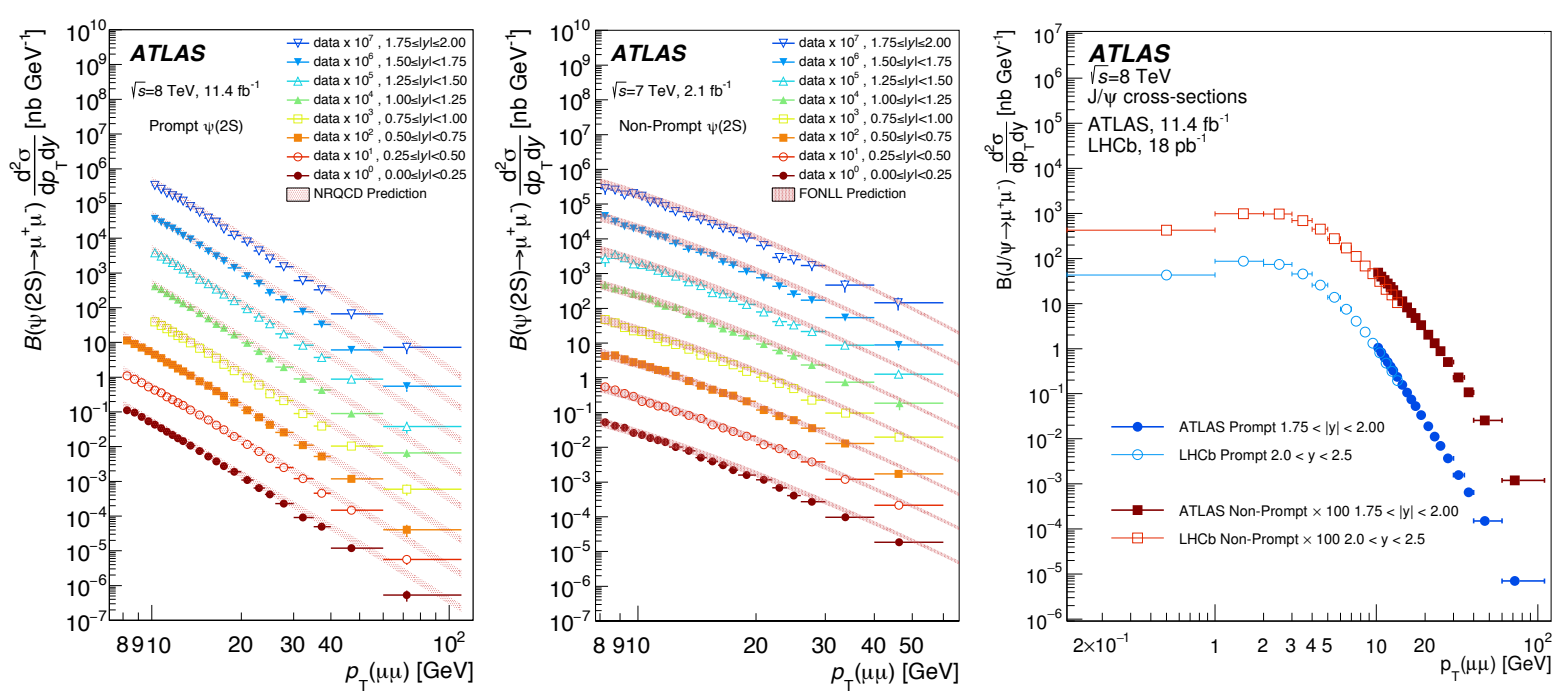

Figure 8: $\psi(2 S)$ prompt and non prompt transverse momentum spectrum, and $J / \psi$ spectra over a wide momentum range, from two measurements. From reference [28].

Figure 8 shows a measurement of the differential $d^{2} \sigma / d p_{\mathrm{T}} d y$ production cross-section for prompt and non-prompt $\psi(2 \mathrm{~S})$, and the comparison between nearby rapidity interval accessible in central (ATLAS in this case) and forward (LHCb) detectors. The measurements are in good agreement with the NRQCD prediction for prompt production, and FONLL for non-prompt production. Note the comparable magnitude and the different $p_{\mathrm{T}}$ dependence of the two production mechanism. The colour-octet LDMEs used in the NRQCD prediction [35] are extracted from a fit to Tevatron data [36]. An apparent continuity in the production behaviour has been observed between $\sqrt{s}=2 \mathrm{TeV}$ studied at the Tevatron and 7, 8, $13 \mathrm{TeV}$ at the LHC.

\subsection{Polarisation of quarkonia}

The polarisation of quarkonia states has attracted attention because of the different predictions provided by the production models. For vector states (e.g. $J / \psi, \psi(2 \mathrm{~S}), \Upsilon(\mathrm{nS}))$ decaying into muon pairs the angular distribution in the rest frame of the decaying particle is described by the parameters $\lambda_{\theta}, \lambda_{\phi}, \lambda_{\theta \phi}[37,38]$. For $p_{\mathrm{T}} \gg m_{Q \bar{Q}}$ the CSM model predicts longitudinal spin-alignment, i.e. $\lambda_{\theta} \simeq-1$, in the quarkonium helicity frame, while NRQCD favours transverse spin-alignment, although different level of polarisation have been predicted $\left(\lambda_{\theta} \simeq 0\right.$ to $\left.+1, \lambda_{\phi} \simeq 0\right)$. The predictions are illustrated in figure 6-right, for $J / \psi \rightarrow \mu^{+} \mu^{-}$, together with a measurement [39].

Similar observations have been performed for $\psi(2 \mathrm{~S})$ and $\Upsilon(\mathrm{nS})[40,41]$. An example is shown in figure 9. The comparison with predictions requires the evaluation of the effects of feed-down from quarkonia states with higher mass. In order to deal with the somewhat arbitrary choice of the reference frame, the data are usually analysed fitting for all parameters of the muons angular distribution, and also considering different choices of frames. Furthermore, combinations of angular distribution parameters that do not depend on the choice of frame have been identified $[37,38]$. So far, the measurements have not shown significant deviations form uniform angular distribution in either the high $p_{\mathrm{T}}$ or the large $y$ regions. 


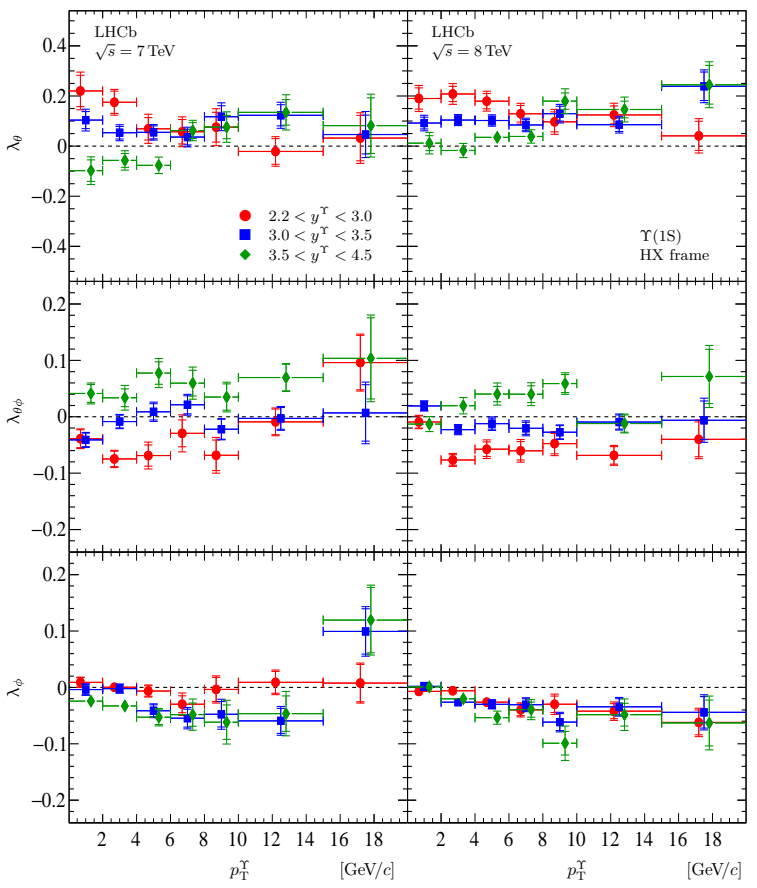

Figure 9: $\Upsilon(1 \mathrm{~S})$ polarisation: the three parameters describing the angular distribution for the decay to muon pairs are shown in $p_{\mathrm{T}}, y$ intervals, in the helicity frame. From reference [41].
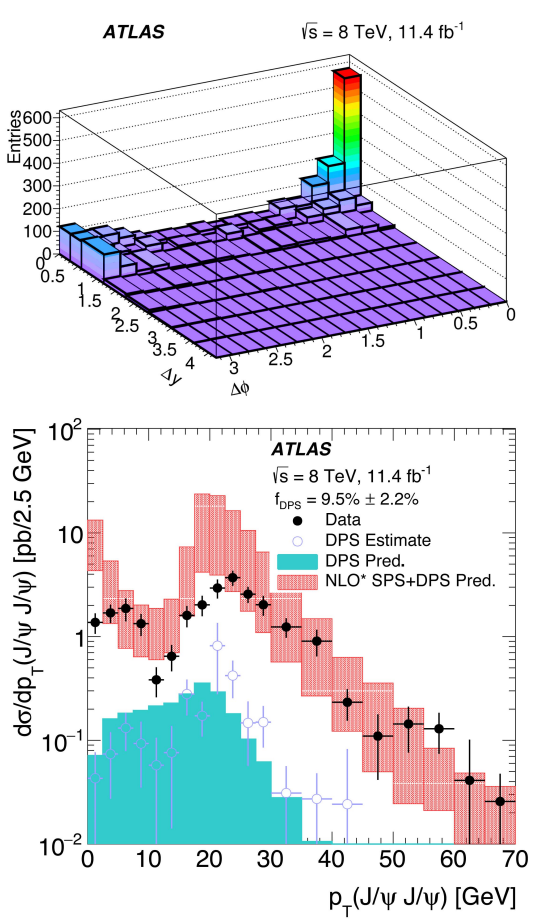

Figure 10: Double prompt $J / \psi$ prompt production. Top: angular separation for SPS component. Bottom: differential cross-section vs. $J / \psi J / \psi$ total transverse momentum. From reference [47].

\subsection{Measurements of associated production}

In recent years the LHC experiments have performed several measurements of associated production, including prompt $J / \psi$ together with $W$ or $Z$, double $J / \psi$, and, most recently, double $\Upsilon$ production, observed by CMS [42]. Recent measurements of double $J / \psi$ production are reviewed here. The interest in these studies lies both in the test of higher order QCD processes [43, 44] in the single parton scattering (SPS), and in the study of double parton scattering (DPS) [45].

LHCb [46] has performed a study where both $J / \psi$ are in the forward $\left(2<y^{J / \psi}<4.5\right)$, low transverse momentum $\left(p_{\mathrm{T}}^{J / \psi}<10 \mathrm{GeV}\right)$ region. The observation is in agreement with the expectations, but does not allow a model independent separation of the SPS and DPS components.

The situation is more favorable in the $\left|y^{J / \psi}\right|<2.1, p_{\mathrm{T}}^{J / \psi}>8.5 \mathrm{GeV}$ region, where ATLAS [47] has used both $\Delta \phi$ and the rapidity separation $\Delta y$ to determine the uncorrelated DPS component. Figure 10 shows the angular separation for the SPS component, peaking at $\Delta y \simeq 0$ and both $\Delta \phi \simeq 0$ and $\simeq \pi$. Also shown is is the production cross-section as a function of the transverse momentum of the pair, which presents as well a bi-modal distribution with a peak at $p_{\mathrm{T}}^{J / \psi J / \psi} \approx 25 \mathrm{GeV}$, in fair agreement with NLO SPS computation. From the measurement of the DPS contribution $\sigma_{\mathrm{DPS}}{ }^{J / \psi J} J / \psi=14.8 \pm 3.8 \mathrm{pb}$ and of the prompt production cross-section $\sigma^{J / \psi}$, the effective cross-section for DPS is derived as $\sigma_{\text {eff }}^{J / \psi J / \psi} \equiv\left(\sigma^{J / \psi}\right)^{2} /\left(2 \times \sigma_{\mathrm{DPS}} J / \psi J / \psi\right)$, and found equal to $6.3 \pm 1.9 \mathrm{mb}$, lower than the values $\approx 15 \mathrm{mb}$ observed for most other DPS processes [47]. 


\section{Conclusions, acknowledgments}

The production of heavy quarks hadrons and quarkonia has been a very active area of studies in recent years, thanks mainly to measurements performed at the LHC and to theory developments in the areas of pertubative and non-relativistic QCD. Correlation in $b-\bar{b}$ production and associated quarkonia production are recently added observables, and others are likely to join in the near future, as new data are being collected.

Sincere thanks to the organisers of HADRON-2017 for the invitation to present this subject, and to Leonid Gladilin, Vakhtang Kartvelishvili, and James Walder for useful discussions.

\section{References}

[1] B.A. Kniehl, G. Kramer, I. Schienbein and H. Spiesberger, Inclusive $D^{* \pm}$ production in $p \bar{p}$ collisions with massive charm quarks, Phys.Rev. D71 (2005) 014018 [hep-ph/0410289]; Collinear subtractions in hadroproduction of heavy quarks, EPJ C41 (2005) 199 [hep-ph/0502194].

[2] M. Cacciari, M. Greco and P. Nason, The $p_{T}$ spectrum in heavy-flavour hadroproduction, JHEP 9805 (1998) 007 [hep-ph/9803400].

[3] T. Sjöstrand, S. Mrenna and Peter Skands, A brief introduction to PYTHIA 8.1, Comput.Phys. Comm. 178 (2008) 852 [hep-ph/0710.3820].

[4] G. Corcella et al., HERWIG 6.5: an event generator for hadron emission reactions with interfering gluons (including supersymmetric processes), JHEP 0101 (2001) 010 [hep-ph/0011363].

[5] S. Frixione, P. Nason and B.R. Webber, Matching NLO QCD and parton showers in heavy flavour production, JHEP 0308 (2003) 007 [hep-ph/0305252].

[6] S. Frixione, P. Nason and G. Ridolfi, A positive-weight next-to-leading-order Monte Carlo for heavy flavour hadroproduction, JHEP 0709 (2007) 126 [hep-ph/0707.3088].

[7] J. Alwall et al., The automated computation of tree-level and next-to-leading order differential cross sections, and their matching to parton shower simulations, JHEP 1407 (2014) 079 [hep-ph/1405.0301].

[8] ATLAS Collaboration, Measurements of the electron and muon inclusive cross-sections in protonproton collisions at $\sqrt{s}=7 \mathrm{TeV}$ with the ATLAS detector, Phys.Lett. B707 (2012) 438 [hep-ex/1109:0525].

[9] LHCb Collaboration, Measurements of prompt charm production cross-sections in pp collisions at $\sqrt{s}$ = 13 TeV, JHEP 1603 (2016) 159, ibid. 1609 (2016) 013, ibid. 1705 (2017) 074 [hep-ex/1510.01707].

[10] M. Cacciari, M.L. Mangano and P. Nason, Gluon PDF constraints from the ratio of forward heavy quark production at the LHC at $\sqrt{s}=7$ and 13 TeV, EPJ C75 (2015) 610 [hep-ph/1507.06197].

[11] CMS Collaboration, Measurement of the total and differential inclusive $B^{+}$hadron cross sections in pp collisions at $\sqrt{s}=13 \mathrm{TeV}$, Phys.Lett. B771 (2017) 435 [hep-ex/1609.00873].

[12] ATLAS Collaboration, Measurement of the differential cross-section of $B^{+}$meson production in pp collisions at $\sqrt{s}=7$ TeV at ATLAS, JHEP 1310 (2013) 042 [hep-ex/1307.0126].

[13] LHCb Collaboration, Measurement of the b-quark production cross-section in 7 and $13 \mathrm{TeV} p p$ collisions, Phys.Rev.Lett. 118, 052002 (2017), ibid. 119, 169901 (2017) [hep-ex/1612.05140]. 
[14] CMS Collaboration, Measurement of the $\Lambda_{b}$ cross section and the $\bar{\Lambda}_{b}$ to $\Lambda_{b}$ ratio with $\Lambda_{b}$ to $J / \psi \Lambda$ decays in pp collisions at $\sqrt{s}=7$ TeV, Phys.Lett. B714 (2012) 136 [hep-ex/1205.0594].

[15] LHCb Collaboration, Study of the production of $\Lambda_{b}^{o}$ and $\bar{B}^{o}$ hadrons in pp collisions and first measurement of the $\Lambda_{b}^{o} \rightarrow J / \psi p K^{-}$branching fraction, Chin.Phys. C40 (2016) 011001 [hep-ex/1509.00292].

[16] CMS Collaboration, Measurement of the ratio of the production cross sections times branching fractions of $B_{c}^{ \pm}$to $J / \psi \pi^{ \pm}$and $B^{ \pm}$to $J / \psi K^{ \pm}$and $\mathscr{B}\left(B_{c}^{ \pm}\right.$to $\left.J / \psi \pi^{ \pm} \pi^{ \pm} \pi^{\mp}\right) / \mathscr{B}\left(B_{c}^{ \pm}\right.$to $\left.J / \psi \pi^{ \pm}\right)$in pp collisions at $\sqrt{s}=7 \mathrm{TeV}, \mathrm{JHEP} 1501$ (2015) 063 [hep-ex/1410.5729].

[17] LHCb Collaboration, Measurement of $B_{c}^{+}$production in proton-proton collisions at $\sqrt{s}=8 \mathrm{TeV}$, Phys.Rev.Lett. 114 (2015) 132001 [hep-ex/1411:2943].

[18] LHCb Collaboration, Study of b $\bar{b}$ correlations in high energy proton-proton collisions, JHEP 1711 (2017) 030 [hep-ex/1708.05994].

[19] ATLAS Collaboration, Measurement of b-hadron pair production with the ATLAS detector in proton-proton collisions at $\sqrt{s}=8 \mathrm{TeV}$, JHEP 1711 (2017) 062 [hep-ex/1705.03374].

[20] H. Fritzsch, Producing heavy quark flavors in hadronic collisions - A test of quantum chromodynamics, Phys.Lett. B67 (1977) 217.

[21] F. Halzen, CVC for gluons and hadroproduction of quark flavours, Phys.Lett. B69 (1977) 105.

[22] ATLAS Collaboration, Measurement of the differential cross-sections of inclusive, prompt and non-prompt $J / \psi$ production in proton-proton collisions at $\sqrt{s}=7 \mathrm{TeV}$, Nucl.Phys. B850 (2011) 387 [hep-ex/1104.3038].

[23] R. Baier and R. Rückl, Hadronic production of $J / \psi$ and $\Upsilon$ : transverse momentum distributions, Phys.Lett. B102 (1981) 364; Hadronic collisions: a quarkonium factory, Z.Phys. C19 (1983) 251.

[24] J. Campbell, F. Maltoni and F. Tramontano, QCD corrections to $J / \psi$ and $\Upsilon$ production at hadron colliders, Phys.Rev.Lett. 98 (2007) 252002 [hep-ph/0703113].

[25] G.T. Bodwin, E. Braaten and G.P. Lepage, Rigorous QCD analysis of inclusive annihilation and production of heavy quarkonium, Phys.Rev. D51 (1995) 1125, ibid. D55 (1997) 5853 [hep-ph/9407339].

[26] LHCb Collaboration, Forward production of $\Upsilon$ mesons in pp collisions at $\sqrt{s}=7$ and 8 TeV, JHEP 1511 (2015) 103; [hep-ex/1509.02372]; Measurement of forward J/ $\psi$ production cross-sections in pp collisions at $\sqrt{s}=13 \mathrm{TeV}$, JHEP 1510 (2015) 172, ibid. 1705 (2017) 063 [hep-ex/1509.00771].

[27] CMS Collaboration, Measurement of the $\Upsilon(1 S), \Upsilon(2 S)$, and $\Upsilon(3 S)$ cross sections in pp collisions at $\sqrt{s}$ = 7 TeV, Phys.Lett. B749 (2015) 14-34 [hep-ex/1303.5900]; Measurement of J/ $\psi$ and $\psi(2 S)$ prompt double-differential cross sections in pp collisions at $\sqrt{s}=7$ TeV, Phys.Rev.Lett. 114 (2015) 191802 [hep-ex/1502.04155].

[28] ATLAS Collaboration, Measurement of the differential cross-sections of prompt and non-prompt production of $J / \psi$ and $\psi(2 S)$ in pp collisions at $\sqrt{s}=7$ and $8 \mathrm{TeV}$ with the ATLAS detector, EPJ C76 (2016) 283 [hep-ex/1512.03657] and BPHY-2012-02 Auxiliary Material.

[29] CMS Collaborations, Measurement of quarkonium production cross sections in pp collisions at $\sqrt{s}=$ $13 \mathrm{TeV}$, hep-ex/1710.11002.

[30] ATLAS Collaboration, Measurements of $\psi(2 S)$ and $X(3872) \rightarrow J / \psi \pi^{+} \pi^{-}$production in pp collisions at $\sqrt{s}=8 \mathrm{TeV}$ with the ATLAS detector, JHEP 1701 (2017) 117 [hep-ex/1610.09303]. 
[31] ATLAS Collaboration, Measurement of $\chi_{c 1}$ and $\chi_{c 2}$ production with $\sqrt{s}=7 \mathrm{TeV}$ pp collisions at ATLAS, JHEP 1407 (2014) 154 [hep-ex/1404.7035].

[32] LHCb Collaboration, Study of $\chi_{b}$ meson production in pp collisions at $\sqrt{s}=7$ and $8 \mathrm{TeV}$ and observation of the decay $\chi_{b} \rightarrow \Upsilon(3 S) \gamma$, EPJ C74 (2014) 3092 [hep-ex/1407.7734]; Measurement of the $\chi_{b}(3 P)$ mass and of the relative rate of $\chi_{b 1}(1 P)$ and $\chi_{b 2}(1 P)$ production, JHEP 1410 (2014) 088 [hep-ex/1409.1408].

[33] CMS Collaboration, Measurement of the production cross section ratio $\sigma\left(\chi_{b 2}(1 P)\right) / \sigma\left(\chi_{b 1}(1 P)\right)$ in $p p$ collisions at $\sqrt{s}=8 \mathrm{TeV}$, Phys.Lett. B743 (2015) 383 [hep-ex/1409.5761].

[34] LHCb Collaboration, Measurement of the $\eta_{c}(1 S)$ production cross-section in proton-proton collisions via the decay $\eta_{c}(1 S) \rightarrow p \bar{p}$, EPJ $\mathbf{C 7 5}$ (2015) 311 [hep-ex/1409.3612].

[35] Y.-Q. Ma, K. Wang, K.-T. Chao, $J / \psi\left(\psi^{\prime}\right)$ production at the Tevatron and LHC at $O\left(\alpha_{s}^{4} v^{4}\right)$ in nonrelativistic QCD, Phys.Rev.Lett. 116 (2011) 042002 [hep-ph/1009.3655].

[36] CDF Collaboration, Measurement of the $J / \psi$ meson and b-hadron production cross sections in $p \bar{p}$ collisions at $\sqrt{s}=1960 \mathrm{GeV}$, Phys.Rev. D71 (2005) 032001 [hep-ex/04112071]; Production of $\psi(2 S)$ mesons in p p collisions at 1.96 TeV, Phys.Rev. D80 (2009) 031103 [hep-ex/0905.1982].

[37] P. Faccioli, C. Lourenço, J. Seixas and H. Wöhri, Towards the experimental clarification of quarkonium polarization, EPJ C69 (2010) 657 [hep-ph/1006.2738].

[38] S. Palestini, Angular distribution and rotations of frame in vector meson decays into lepton pairs, Phys.Rev. D83 (2011) 031503 [hep-ph/1012.2485].

[39] LHCb Collaboration, Measurement of J/ $\psi$ polarization in pp collisions at $\sqrt{s}=7 \mathrm{TeV}, \mathrm{EPJ} \mathbf{C 7 3}$ (2013) 2631 [hep-ex/1307.6379].

[40] CMS Collaboration, Measurement of the $\Upsilon(1 S), \Upsilon(2 S)$, and $\Upsilon(3 S)$ polarizations in pp collisions at $\sqrt{s}$ $=7$ TeV, Phys.Rev.Lett. 110 (2013) 081802 [hep-ex/1209.2922]; Measurement of the prompt J/ $\psi$ and $\psi(2 S)$ polarizations in pp collisions at $\sqrt{s}=7$ TeV, Phys.Lett. B727 (2013) 381 [hep-ex/1307.6070]; $\Upsilon(n S)$ polarizations versus particle multiplicity in pp collisions at $\sqrt{s}=7 \mathrm{TeV}$, Phys.Lett. B761 (2016) 31 [hep-ex/1603.02913].

[41] LHCb Collaboration, Measurement of the $\Upsilon$ polarization in pp collisions at $\sqrt{s}=7$ and $8 \mathrm{TeV}$, hep-ex/1709.01301.

[42] CMS Collaboration, Observation of $\Upsilon(1 S)$ pair production in proton-proton collisions at $\sqrt{s}=8 \mathrm{TeV}$, JHEP 1705 (2017) 013 [hep-ex/1610.07095].

[43] J.-Ph. Lansberg and H.-S. Shao, Production of $J / \psi+\eta_{c}$ versus $J / \psi+J / \psi$ at the LHC: Importance of real $\alpha_{s}^{5}$ corrections, Phys.Rev.Lett. 111 (2013) 122001 [hep-ph/1308.0474].

[44] L.-P. Sun, H. Han and K.-T. Chao, Impact of J/ $\psi$ pair production at the LHC and predictions in nonrelativistic QCD, Phys.Rev. D94 (2016) 074033 [hep-ph/1404.4042].

[45] C.H. Kom, A. Kulesza and W.J. Stirling, Pair production of $J / \psi$ as a probe of double parton scattering at LHCb, Phys.Rev.Lett. 107 (2011) 082002 [hep-ph/1105.4186].

[46] LHCb Collaboration, Measurement of the $J / \psi$ pair production cross-section in pp collisions at $\sqrt{s}=$ 13 TeV, JHEP 1706 (2017) 047, ibid. 1710 (2017) 068 [hep-ex/1612.07451].

[47] ATLAS Collaboration, Measurement of the prompt $J / \psi$ pair production cross-section in pp collisions at $\sqrt{s}=8$ TeV with the ATLAS detector, EPJ C77 (2017) 76 [hep-ex/1612.02950]. 